\title{
Optical Properties of Polyvinyl Alcohol Films Modified with Silver Nanoparticles
}

\author{
Vladimir Agabekov, ${ }^{1}$ Nadezhda Ivanova, ${ }^{1}$ Viacheslav Dlugunovich, ${ }^{2}$ and Igor Vostchula ${ }^{2}$ \\ ${ }^{1}$ Institute of Chemistry of New Materials, The National Academy of Sciences of Belarus, F. Skarina Street 36, 220141 Minsk, Belarus \\ ${ }^{2}$ B. I. Stepanov Institute of Physics, The National Academy of Sciences of Belarus, Nezalezhnastsi Ave. 68, 220072 Minsk, Belarus
}

Correspondence should be addressed to Vladimir Agabekov, agabekov@ichnm.basnet.by

Received 29 December 2011; Accepted 25 January 2012

Academic Editor: Sevan P. Davtyan

Copyright ( 2012 Vladimir Agabekov et al. This is an open access article distributed under the Creative Commons Attribution License, which permits unrestricted use, distribution, and reproduction in any medium, provided the original work is properly cited.

Polyvinyl alcohol (PVA) films modified with Ag nanoparticles and orientated by single-axial tension do not possess dichroism at light passing as well as the film that is cross-linked under the action of bichromates and UV light. These films are double-ray refracting and behave themselves as phase plates that transform linearly polarized incident radiation to elliptically polarized one. The film of latter type has optical properties of reflective diffuse polarizer with polarizing ability $\sim 0.8$ in the range of observation angles from $20^{\circ}$ to $80^{\circ}$.

\section{Introduction}

The influence of the addition of nanoparticles of various types on the properties of polymers is intensively investigated lately since introduction of even a small amount of nanoparticles to the polymeric matrix can strongly change mechanical, thermal, optic, and other properties of a material [1]. It is known that for manufacturing of polymer films with high light and thermal stability they are modified with silver nanoparticles $[2,3]$, and to improve moisture resistance of polymer matrices they are treated with strong oxidants that are cross-linked PVA molecules under the influence of ultraviolet irradiation [4].

PVA films modified with Ag nanoparticles may be used as linear film polarizers, compensative retarder films and diffusely scattering elements for creation and control of illumination background of liquid crystal displays (LCD) operating in twilight illumination and also in sunlight as well as in the devices of latent pattern identification.

We have developed polaroid films of transmissive, reflective $[5,6]$, and transflective types, including those with extended temperature range of operation [7] for the nearIR [8] and UV [9] regions, as well as a new generation of circular and reflective linear polarizers with improved optical characteristics.
The purpose of this work is to examine the ability of PVA films modified with silver nanoparticles, to scatter incident linear polarized He-Ne laser light, as well as to determine the effect of processing conditions (uniaxial tension, cross-linking of the polymer matrix during irradiation by UV radiation in presence of bi-chromates) on scattering and polarizing properties of modified PVA films.

\section{Experimental Procedure}

The solution of PVA "Mowiol 28-99" of Hoechst Akiengesllschaft (Germany) (10 wt \%) with characteristics presented in Table 1 was used to obtain PVA films.

Dosed amounts of modifier, solution $\mathrm{AgNO}_{3}$ (2 wt \%) and glycerin $(2.3-2.8 \mathrm{wt} \%)$ as plasticizer and weak reducer that were dispersed by ultrasound from $30 \mathrm{~min}$ to a homogeneous consistence, were incorporated into a cooled solution of PVA to modify PVA films with Ag nanoparticles. After that watering solutions were applied on a degreased surface using a filler of a smearing type. Liquid films were dried in the drying chamber at $35 \pm 5^{\circ} \mathrm{C}$ in solvent atmosphere during 1 day up to residual humidity $7-10 \%$. Oxidation-reduction reaction of $\mathrm{Ag}^{+}$and glycerin with the formation of silver nanoparticles in colloid stage occurs inside the films at drying, and these nanoparticles give orange-brown color to 
TABLE 1: Characteristics of polyvinyl alcohol.

\begin{tabular}{|c|c|c|c|c|c|}
\hline PVA & Molecular weight & Polymerization degree & Content of acetate groups, $\%$ & Solubility, \% & Transparency, \% \\
\hline Mowiol 28-99 & 145000 & 3300 & 0.6 & 99.9 & 94.6 \\
\hline
\end{tabular}

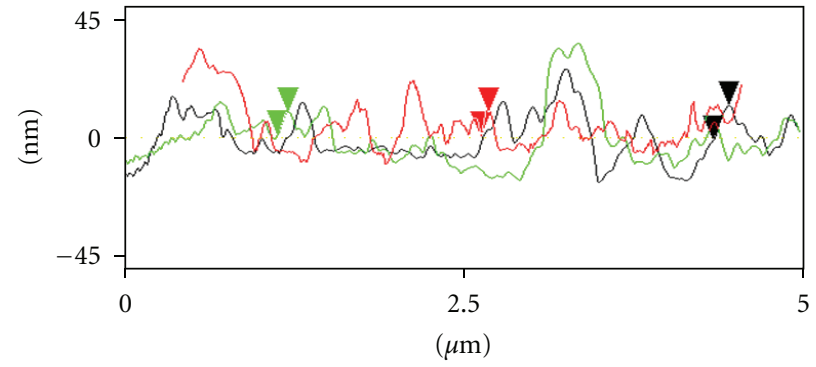

(a)

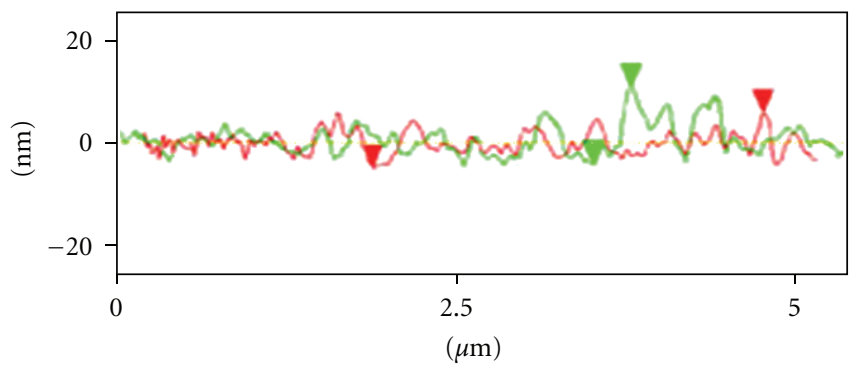

(b)

FIgURE 1: Atomic force microscope surface images and profile of PVA films modified with Ag nanoparticles: (a) nonoriented film; (b) oriented and cross-linked PVA film.

the films. $60 \mathrm{~nm}$ thick modified films containing $0.6 \mathrm{wt} \%$ of silver nanoparticles, that had maximal optical density at the wave length 410-420 nm and particle size 10-20 nm (according to [10]), were chosen for studies. It has been pointed out in article [11] that the adsorption spectrum of silver nanoparticles in colloidal solution having one narrow and permitted (like in our case) peak, that is localized at $\lambda=$ $410 \mathrm{~nm}$, indicates the monodispersity and uniformity of separate silver nanoparticles of a spherical shape. The surface roughness of investigated films ranged from 10 to $20 \mathrm{~nm}$ (Figure 1(a)). The roughness of films surface considerable decreased to $3-10 \mathrm{~nm}$ after single-axial orientation with axial ratio 4 and cross-linking under the action of oxidizerammonium bichromate with following UV radiation (Figure 1(b)). The degree of cross-linking of PVA molecules in the film was controlled by UV spectra of reflectance (Fourier UV-spectrophotometer "Nicolet"-Protégé-460)the disappearance of intensive strip $3340 \mathrm{~cm}^{-1}$ that is related to valent fluctuations of $\mathrm{OH}$ group.

Scattering properties of PVA films modified with Ag nanoparticles and polarization characteristics of reflected and transmitted radiation were investigated using laser goniophotometric Stokes polarimeter [12]. Radiation of He-Ne laser with $0.63 \mu \mathrm{m}$ wavelength and $5 \mathrm{~mm}$ beam diameter was directed at an angle of $\sim 5^{\circ}$ to the film surface normal. Analogous lightning conditions are often realized in practical use of LCD. Radiation reflected by films was recorded at the observation angles $(\beta)$ ranging from $5^{\circ}$ to $80^{\circ}$ and characteristics of radiation transmitted by a film were recorded at registration angle $175^{\circ}$ to the surface normal. Solid angle, where the radiation was registered, was $7.2 \cdot 10^{-3} \mathrm{sr}$, which conformed to angular resolution in the plane of incidence. The registration plane was situated horizontally and coincided with the incident plane. Incident radiation was polarized in the registration plane or cross-polarized.

Polarization characteristics of radiation as a degree of polarization $(P)$, azimuth of polarization $(\varphi)$, and polarization ellipticity $(e)$ were determined on the basis of measured of Stokes parameters $\left(I, P_{1}, P_{2}, P_{3}\right)$ of radiation reflected by investigated films [13]

$$
\begin{gathered}
P=\frac{\sqrt{P_{1}^{2}+P_{2}^{2}+P_{3}^{2}}}{I}, \\
e=\frac{\sqrt{P_{1}^{2}+P_{2}^{2}+P_{3}^{2}}-\sqrt{P_{1}^{2}+P_{2}^{2}}}{P_{3}} \\
\varphi=\frac{1}{2} \arctan \frac{P_{2}}{P_{1}}
\end{gathered}
$$

where $I$ is the total intensity of radiation reflected within the solid angle of the registration system, $P_{1}$ is the parameter that characterizes the advantage of horizontal polymerization, $P_{2}$ is the parameter that characterizes the advantage of linear polarization with $45^{\circ}$ azimuth, and $P_{3}$ is the parameter that characterizes the advantage of right-circular polarization. All Stokes parameters $\left(I, P_{1}, P_{2}, P_{3}\right)$ have units of intensity and completely characterize the polarization state of radiant flux. 


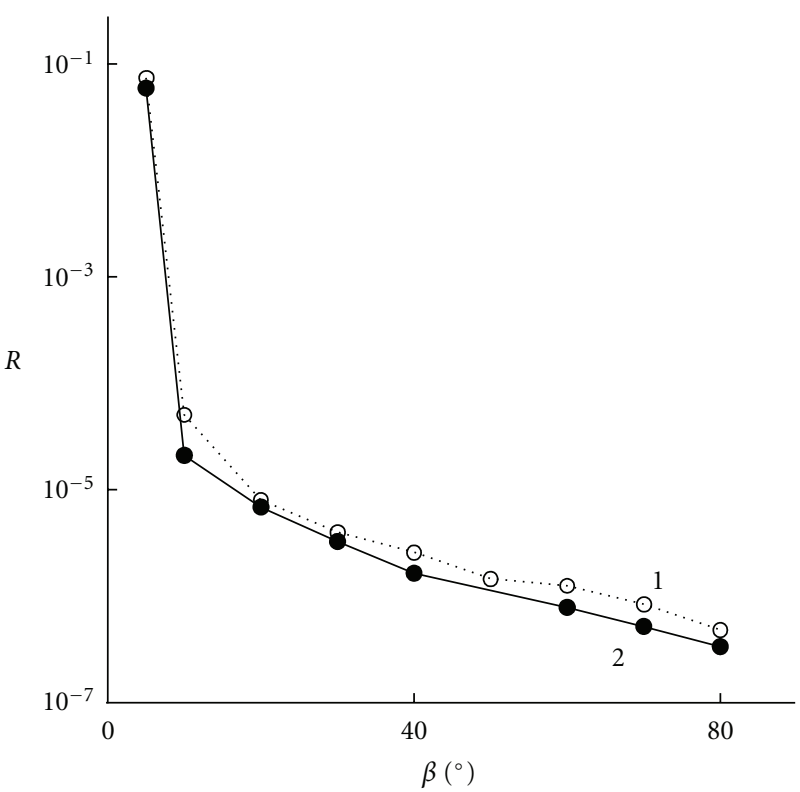

(a)

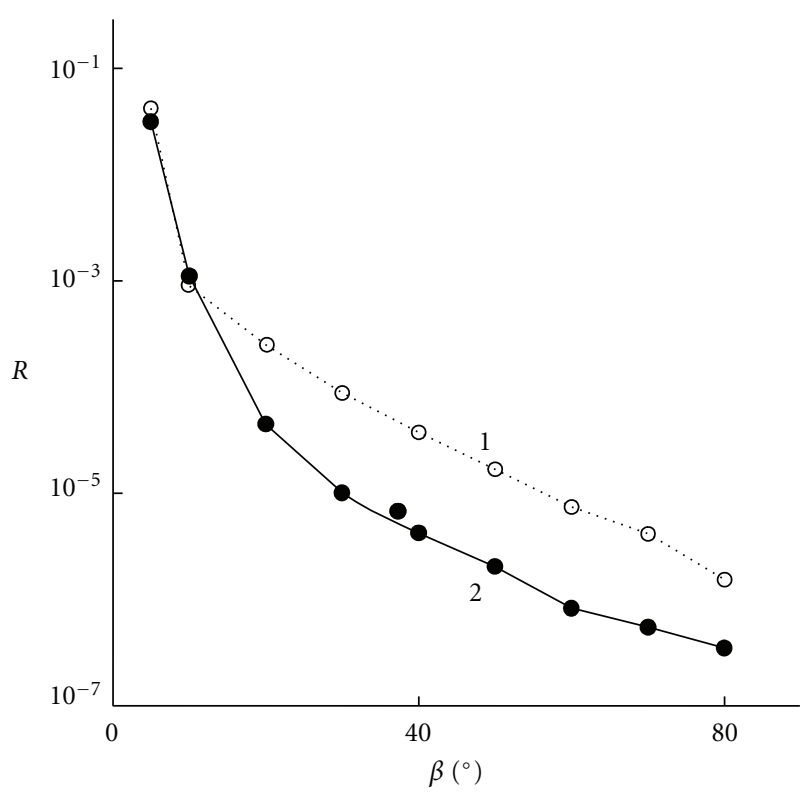

(b)

FIGURE 2: The dependence of bidirectional reflectance coefficient of PVA films modified by Ag nanoparticles on the angle of reflection: (a) only oriented film; (b) oriented and cross-linked film. Incident radiation is polarized: (1) in plane of incidence, (2) perpendicular to the plane of incidence.

Bidirectional reflectance $R(\alpha, \beta)$, transmittance $T(\alpha, \beta)$, and polarizing efficiency $q(\alpha, \beta)$ of investigated films were determined by the following equations:

$$
\begin{gathered}
R(\alpha, \beta)=\frac{I_{r}}{I_{0}}, \\
T(\alpha, \beta)=\frac{I_{t}}{I_{0}}, \\
q(\alpha, \beta)=\frac{R_{\max }-R_{\min }}{R_{\max }+R_{\min }},
\end{gathered}
$$

where $I_{r}, I_{t}$, and $I_{0}$ are the intensities of reflected, transmitted, and incident radiation, respectively; $\alpha$ and $\beta$ are the incident and registration angles; $R_{\max }$ and $R_{\min }$ are bidirectional reflectances of film illuminated with radiation linearly coand cross-polarized to the film strain direction, respectively. Relative standard deviation of the measurement of bidirectional reflectance, transmittance, and degree of polarization did not exceed 0.05 .

\section{Results and Discussion}

PVA films modified with Ag nanoparticles specularly reflect incident radiation (Figure 2).

Bidirectional specular reflectance coefficient of modified and oriented film in the direction of specular reflection is $\sim 0.07$. With the increase of observation angle up to $15^{\circ} \mathrm{R}$ values sharply decrease and do not depend on the azimuth of polarization of the incident radiation (Figure 2(a)). During further increase of $\beta$, the values of bidirectional reflectance coefficients $(R)$ of investigated film decrease substantially slower and monotonously. Hence, reflection from the investigated film is diffuse at registration angle more than $15^{\circ}$. Besides that, difference in Fresnel reflectance of investigated material manifests its effect in diffusely reflected flux [14]. This shows that, at sounding with radiation that is polarized orthogonally to the plane of incidence, $R(\alpha, \beta)$ values of investigated samples are systematically higher than in case of illumination by copolarized laser radiation (Figure 2(a)).

For PVA film modified with Ag nanoparticles, oriented and cross-linked with oxidizers under UV radiation, the difference in reflectance coefficients for orthogonally linear polarized components of sounding radiation at observation angles increasing from $20^{\circ}$ to $80^{\circ}$ reaches one order of magnitude. This means that the given film is a reflecting linear polarizer with polarizing ability $q \approx 0.8$ in the range of observation angles from $20^{\circ}$ to $80^{\circ}$ (Figure 3 ) and angular distribution of reflected radiation close to a diffuse one (Figure 2(b)). At the same time its bidirectional reflection coefficients is on nearly two orders of magnitude higher than $R(\alpha, \beta)$ of just oriented PVA film (Figures $2(\mathrm{a})$ and 2(b), curve 1).

When comparing polarization characteristics of radiation, scattered by modified films, it can be seen that at observation angles from $5^{\circ}$ to $40^{\circ}$ the degree of polarization $P$ of reflected radiation is equally high and is $0.8 \div 1.0$ for both the simply oriented film (Figure $4(\mathrm{a})$ ) and the cross-linked film (Figure 4(b)) and does not depend on the azimuth of linearly polarized radiation.

The change of the degree of polarization of radiation scattered by modified PVA films at the reflected angles exceeding $40^{\circ}$ depends on polarization azimuth of sounding radiation. Thus, if the film was illuminated with radiation 


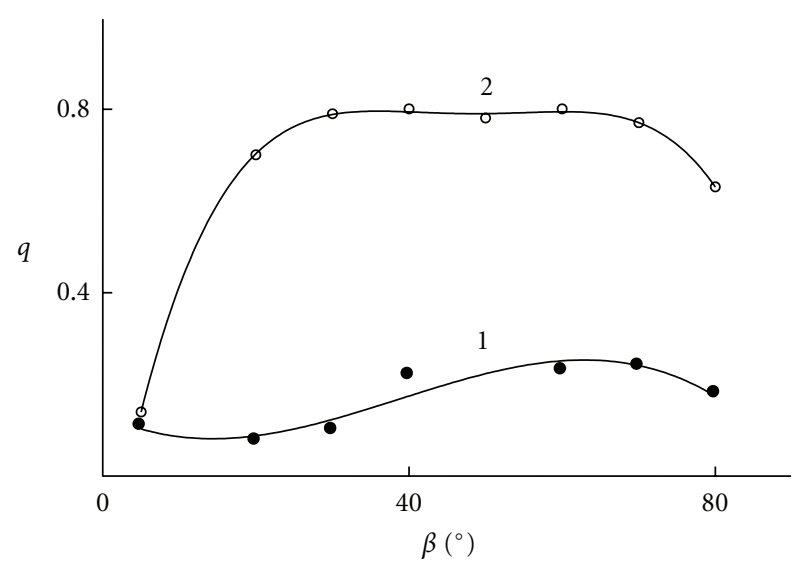

Figure 3: Polarizing efficiency of PVA films modified with Ag nanoparticles: (1) only oriented film; (2) oriented and cross-linked film.

that is polarized perpendicular to the plane of incidence, the degree of polarization of radiation scattered by it remains high-no, less than 0.8 at the increase of the reflected angle up to $80^{\circ}$ (Figures $4(\mathrm{a})$ and $4(\mathrm{~b})$ ). High values of the degree of polarization of radiation, scattered by films over the whole range of observation angles (from $5^{\circ}$ up to $80^{\circ}$ ), testify the preservation of polarization state of sounding radiation, which is polarized perpendicular to the plane of incidence. This is verified by the results of measurement of azimuth of polarization and ellipticity of radiation scattered by modified PVA films at mentioned sounding conditions. Indeed, the azimuths of polarization of scattered and sounding radiations coincide, and there is no elliptically polarized component in the scattered flux.

The degree of polarization of radiation scattered by investigated films illuminated with co-polarized radiation is monotonously decreased to 0.6 with the increase of reflected angle up to $80^{\circ}$ (Figures $4(\mathrm{a})$ and $4(\mathrm{~b})$ ).

The results of measurement of bidirectional transmittance coefficients of investigated PVA films illuminated with radiation at $\alpha=5^{\circ}$ linearly polarized in the plane of incidence $\left(T_{\|}\right)$as well as in a plane that is orthogonal to it $\left(T_{\perp}\right)$ and also with azimuth $45^{\circ}\left(T_{45}\right)$ in relation to the direction of film stretching and polarization characteristics of transmitted radiation (degree of polarization, ellipticity, and azimuth of polarization) at registration angle of $175^{\circ}$ are presented in Table 2.

It follows from the presented data that bidirectional transmittance coefficients of investigated modified PVA films are practically the same for orthogonally linear polarized radiation components. This indicates that there is no amplitude anisotropy for transmitted radiation in investigated films, and they are not linear polarizers. At the same time, investigated films possess phase anisotropy that occurs because of orientation of films by stretching and behaving themselves as phase plates.

The radiation that is linearly polarized at the angle of $45^{\circ}$ to the film orientation direction is transformed in elliptically polarized radiation when transmitted through oriented

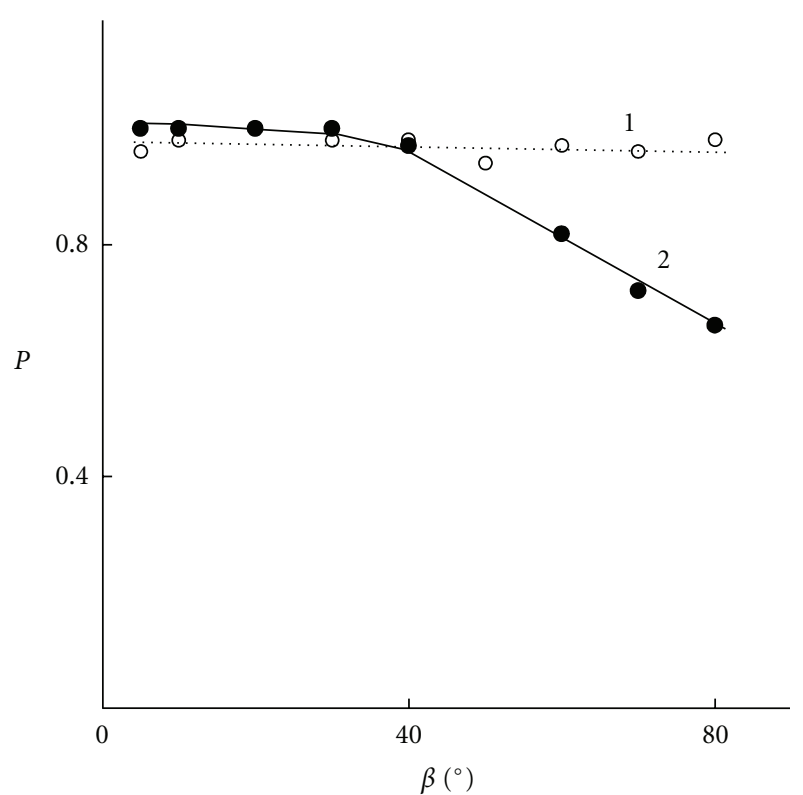

(a)

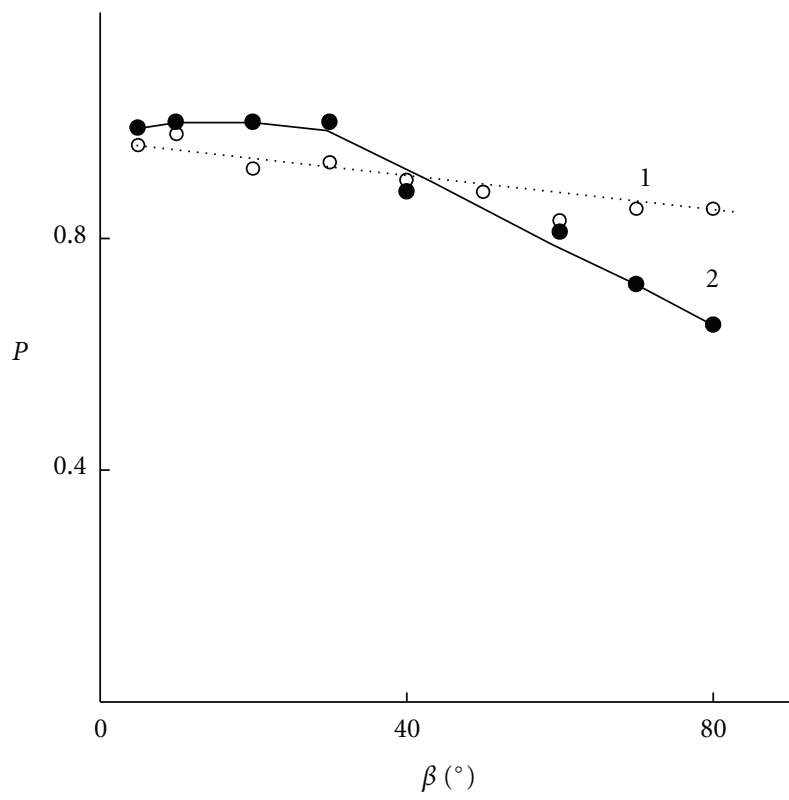

(b)

FIGURE 4: The dependence of the degree of polarization of radiation reflected by modified PVA films on reflection angle: (a) only oriented film; (b) oriented and cross-linked film; radiation is copolarized (1) and cross-polarized (2).

modified PVA films. This phase anisotropy can be used for compensation of undesirable phase delay in radiation components that appear during passing of radiation through different elements of LCD.

\section{Conclusions}

PVA film modified with Ag nanoparticles (0.6 wt\%) that is oriented and cross-linked under the action of oxidizers 
TABle 2: Optical parameters of modified PVA films and polarization characteristics of transmitted radiation at registration angle of $175^{\circ}$.

\begin{tabular}{lcc}
\hline $\begin{array}{l}\text { Type of treatment } \\
\text { characteristics }\end{array}$ & Oriented PVA film & $\begin{array}{c}\text { Oriented and } \\
\text { cross-linked PVA film }\end{array}$ \\
\hline$T_{\|}$ & 0.73 & 0.44 \\
$T_{\perp}$ & 0.78 & 0.51 \\
$T_{45}$ & 0.77 & 0.52 \\
$P_{\|}$ & $0.99 \pm 0.01$ & $1.00 \pm 0.01$ \\
$P_{\perp}$ & $0.97 \pm 0.01$ & $0.98 \pm 0.01$ \\
$P_{45}$ & $0.96 \pm 0.01$ & $0.89 \pm 0.01$ \\
$e_{\|}$ & $0.01 \pm 0.01$ & $0.01 \pm 0.01$ \\
$e_{\perp}$ & $-0.01 \pm 0.01$ & $0,03 \pm 0.02$ \\
$e_{45}$ & $0.02 \pm 0.02$ & $0.81 \pm 0.04$ \\
$\varphi_{\|}$, degrees & $-3.8 \pm 0.03$ & $1.4 \pm 0.2$ \\
$\varphi_{\perp}$, degrees & $88.0 \pm 1.3$ & $86.4 \pm 0.2$ \\
$\varphi_{45}$, degrees & $-28.9 \pm 0.07$ & $100.0 \pm 1.0$ \\
\hline
\end{tabular}

and UV-radiation, can be used as reflective diffuse linear polarizer with polarizing ability $\sim 0.8$ in the range of observation angles from $20^{\circ}$ to $80^{\circ}$.

It has been established that investigated films do not possess amplitude anisotropy (dichroism) when transmitting the light, but do possess phase anisotropy (birefringence) which depends on nanoparticles concentration and film thickness and they behave themselves as uniaxial phase plates that transform linearly polarized incident radiation into elliptically polarized radiation.

\section{References}

[1] W. A. Goddard, D. W. Brenner, S. E. Lyshevski, and G. J. Lafrate, Handbook of Nanoscience, Engineering, and Technology, CRC Press, London, UK, 2003.

[2] V. I. Studenov and M. G. Tomilin, "Heat- and moisture-resistant thin-film polarizer," Journal of Optical Technology, vol. 66, no. 6, pp. 550-553, 1999.

[3] V. E. Agabekov, N. A. Ivanova, E. V. Karpinchik, T. G. Kosmacheva, V. A. Dlugunovich, and O. V. Tsarukb, "Polarizing properties of polymeric films modified by silver nanoparticles," in Lasers for Measurements and Information Transfer, vol. 7006 of Proceedings of SPIE, June 2008.

[4] A. Emdin, "Polyvinyl alcohol—polymer-based copy layer," Printing, no. 1, pp. 38-40, 1977.

[5] V. E. Agabekov, N. A. Ivanova, V. V. Daineko et al., "Method of forming reflective coating for thin-film polarizers, reflective type," Patent RB no. 4678, 2002.

[6] V. E. Agabekov, N. A. Ivanova, I. V. Kulevskaya et al., "Composition for reflective coating on the polarizers," Patent RB no. 12643, 2009.

[7] V. E. Agabekov, N. G. Ariko, and N. A. Ivanova, "Film polarizers for liquid crystal display units," Vestsi NAS B, Seria Himicheskih Navuk, vol. 4, pp. 98-112, 2002.

[8] N. A. Ivanova, V. E. Agabekov, O. A. Daineko, I. Yu. Globa, and O. V. Tsaryuk, "Polarizing film for near-infrared spectrum," Vestnik BGUIR, vol. 35, no. 5, pp. 77-85, 2008.

[9] L. N. Filipovich, N. G. Ariko, and V. E. Agabekov, "Spectral properties and light fastness of the polarization of the films, painted brilliant yellow," Journal of Applied Spectroscopy, vol. 76, no. 3, pp. 460-466, 2009.

[10] B. G. Ershov and N. L. Sukhov, "Linear clusters of silver in water solution (clusterites): radiation-chemical synthesis and their properties," Izvestia RAN, no. 6, pp. 1429-1434, 1996.

[11] B. H. Choi, H. H. Lee, S. Jin, S. Chun, and S. H. Kim, "Characterization of the optical properties of silver nanoparticle films," Nanotechnology, vol. 18, no. 7, Article ID 075706, 2007.

[12] V. A. Dlugunovich, L. V. Simonchik, V. N. Snopko, and O. V. Tsaryuk, "Goniophotometric laser stokes-polarimeter," Measurement Techniques, vol. 43, no. 1, pp. 31-37, 2000.

[13] V. N. Snopko, Polarization Characteristics of Optical Radiation and Their Measurement Methods, Nauka i tehnika, Minsk, Belarus, 1992.

[14] M. Born and E. Wolf, The Basics of Optics, Nauka, Moscow, Russia, 1973. 

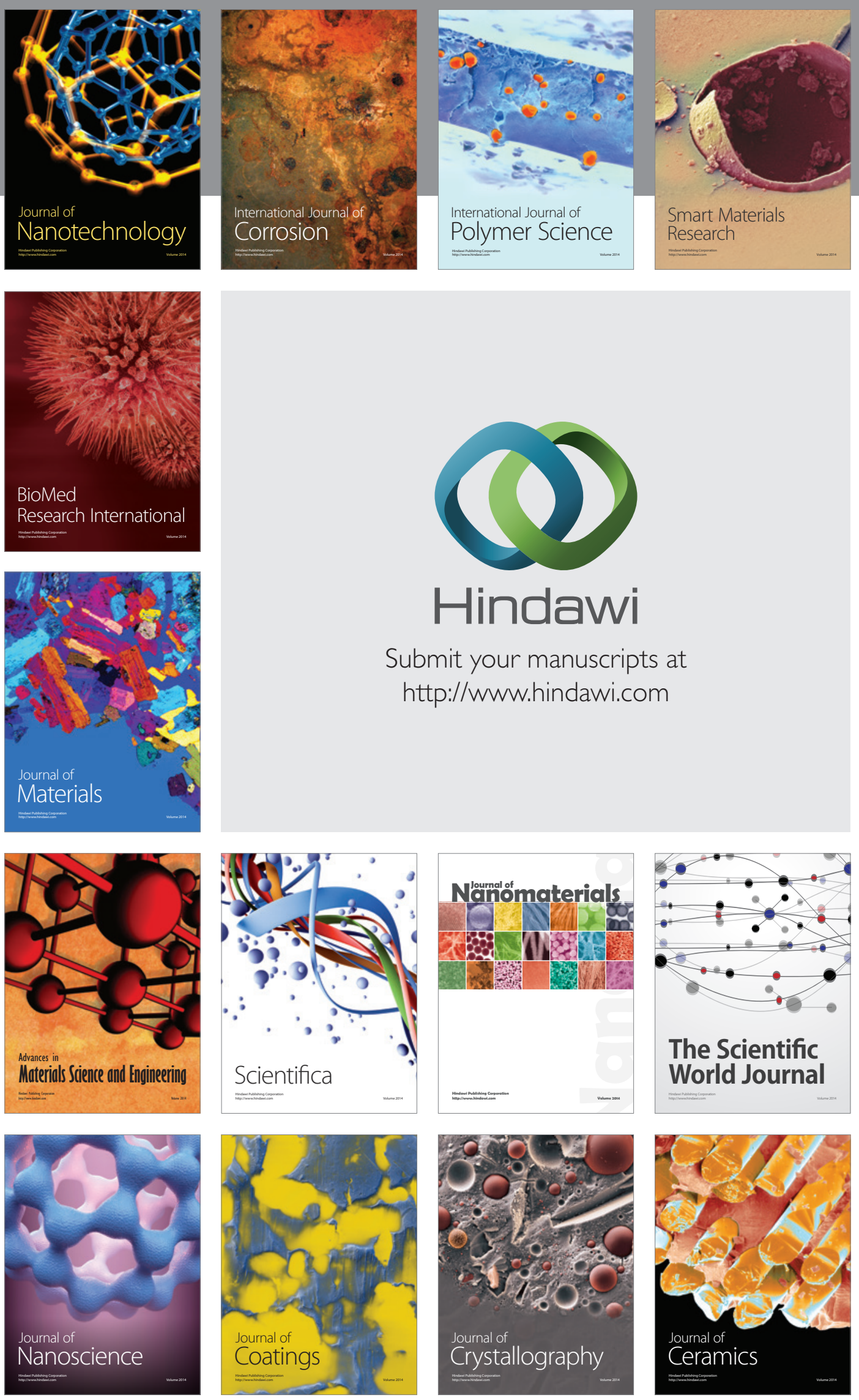

The Scientific World Journal

Submit your manuscripts at

http://www.hindawi.com

\section{World Journal}

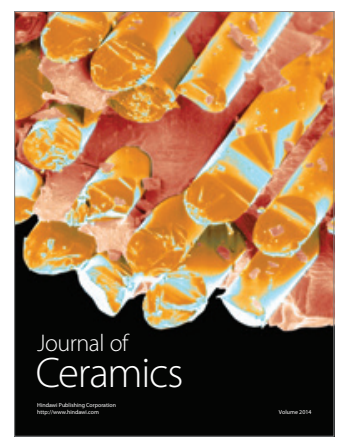

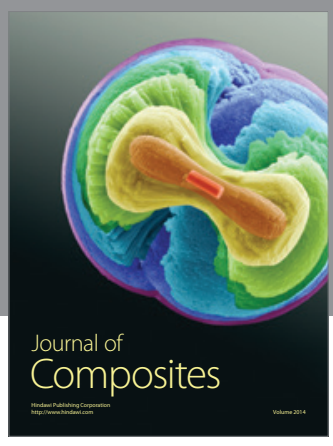
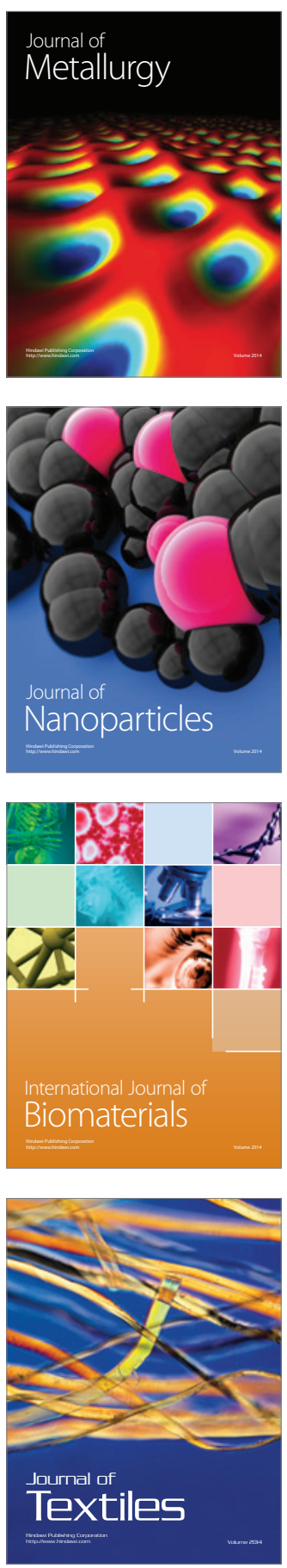